\title{
Búsqueda de descriptores que caractericen una disciplina emergente en WoS y SCOPUS: el caso de la Educación Matemática
}

\author{
Natividad Adamuz-Povedano \\ Noelia Jiménez-Fanjul \\ Alexander Maz-Machado
}

Universidad de Córdoba - UCO, España

ARTÍCULO / ARTICLE

\begin{abstract}
Resumen
Con la complejidad que van adquiriendo ciertas disciplinas al tener conexiones y relaciones con otras ramas del conocimiento de las que reciben aportes, se hace necesario identificar cuáles son los descriptores que caractericen de forma específica su producción científica. Tomamos como ejemplo prototipo a la Educación Matemática por la existencia de ambigüedad de criterio en la concreción de los términos clave para la producción científica de esta disciplina. Todo esto hace pues necesario el establecimiento de un listado de descriptores que la caracterice. Presentamos un desarrollo metodológico para obtener un listado de descriptores básicos de la producción científica publicados en revistas científicas indexadas en las bases de datos de la Web of Science (WoS) y SCOPUS durante un periodo de 30 años, de 1980 a 2009, para esta disciplina.
\end{abstract}

Palabras clave

Educación matemática ; Descriptores ; Bibliometría ; Producción científica ; Recuperación de la información

Search for descriptors that characterize an emerging discipline in WoS and SCOPUS: the case of mathematics education

\begin{abstract}
Some social sciences become very complex due to de links and specific connexions with some others disciplines. This fact explains the necessity of identifying which are the main descriptors or keywords that characterize unequivocally their scientific production. One example of this is the case of mathematics education. This case is also special due to the existing ambiguity in the identification of validated keywords for it. Thus, it becomes necessary to establish a list of descriptors that characterize a research article as a Mathematics Education one. We present a methodology to obtain a core descriptors list for accessing to the Mathematic Education articles published in journals indexed by both the Web of Sciences (WoS) and SCOPUS databases during a period of time of 30 years, since 1980 up to 2009 .
\end{abstract}

Keywords

Mathematics education ; Keywords ; Bibliometrics ; Scientific production ; Information retrieval 


\section{Introducción}

Cuando alguien se inicia en la investigación de cualquier campo científico, lo primero que debe hacer es conocer los fundamentos teóricos del campo y familiarizarse con los descriptores tanto generales, como específicos de su tópico de interés dentro del campo. El conocer cuáles son los documentos de referencia es condición necesaria para abordar la solución a un problema que se plantee (Sanz-Valero et al., 2008). En los programas de doctorado es común que en las asignaturas de metodología de la investigación se den pautas para realizar búsquedas bibliográficas en las bases de datos.

Como afirma Barité (2009, p. 15) "La documentación de una especialidad es, naturalmente, la que provee la terminología más actual, representativa y ajustada al lenguaje de los usuarios". La necesidad de contar con una lista básica de descriptores hace que cobre importancia debido al continuo aumento de revistas científicas, y por tanto, de la información. Esta eclosión de publicaciones hace cada vez más difícil realizar búsquedas manuales y por tanto debe recurrirse a los buscadores informáticos. En las ciencias y disciplinas consolidadas los tesauros ofrecen los descriptores específicos que permiten recuperar la información sobre un tema determinado. Pero muchas veces en disciplinas jóvenes o aquellas que reciben aportes desde distintos ámbitos científicos no se dispone de tesauros que ayuden en esta tarea. Entre ellas se encuentran las didácticas específicas.

Ante esta situación, algunas revistas de Educación no exigen que los descriptores correspondan a un determinado tesauro o bien recurren al Tesauro Europeo de Educación (TEE) o al Tesauro de ERIC (Education Resources Information Center). Esto último hace que los autores utilicen descriptores genéricos de Educación lo que lleva a que cuando se realizan búsquedas sobre ciertas temáticas de las didácticas específicas sus trabajos no sean recuperados. Otra variante que se pone de moda, es lo que se denomina como Folksonomía (Rodríguez, 2009; Strehl, 2011), que se muestra como una alternativa entre los lenguajes documentales. Esto es una clasificación social que se crea por agregación de información sin ningún punto de partida previo (Quintarelli, 2005), generándose así una serie de descriptores definidos de forma subjetiva y a criterio del autor la determinación de si el término es adecuado o no. Esta libertad de descriptores afecta no solo a la visibilidad de los autores, sino que también sus aportes en su campo de investigación se diluyen en el ingente volumen de información de la investigación educativa.

El proceso de búsqueda exhaustiva de las fuentes relevantes sobre temas determinados es una actividad larga y compleja, si el tema está relacionado con varias disciplinas (Alcain y Ruíz-Galvez, 1996), como sucede con las didácticas específicas. Al respecto se han propuesto algunas metodologías para la estructuración de este tipo de disciplinas con limites difusos respecto a otras (Senso, Magaña-Redondo, Faber-Benitez y Vila-Miranda, 2007; Marín, 2010; Granados y Nicolau, 2007).

Vamos a seguir una estrategia metodológica para recuperar la producción específica de estas disciplinas. Tomaremos como prototipo a la Educación Matemática (EMA). Esta recibe aportaciones de diversas disciplinas como Matemáticas, Psicología, Sociología, Pedagogía, Epistemología y Filosofía de las matemáticas, Lingüística, etc. (Godino, 1991) porque la misma problemática no sólo afecta a las publicaciones sino también a otro tipo de búsquedas de la producción científica, como son las tesis doctorales en lois repositorios y bases de datos. A modo de ejemplo, hemos consultado en la base de datos española de tesis doctorales TESEO los descriptores de las tesis doctorales de EMA, el listado de las tesis se tomado de los datos utilizados en investigaciones previas (Autor, 2012a; Autor, 2003). Se halló que de los 1092 descriptores solamente 11\% son específicos de la Educación Matemática, los demás son genéricos de educación.

Hasta ahora los estudios bibliométricos realizados sobre EMA, han utilizado fuentes bibliográficas de las que se tenía la certeza de que pertenecían a la disciplina, a saber: tesis doctorales realizadas en departamentos de didáctica de las matemáticas, congresos o revistas especificas de Educación Matemática (Autor, 2012a; Autor, 2011; Autor, 2009), pero no hay registro en la literatura científica de investigaciones sobre esta disciplina tomando como fuentes las bases de datos internacionales que indexan todo tipo de revistas susceptibles de publicar artículos de Educación Matemática aunque esas revistas no sean exclusivas de la disciplina. Si se ha realizado un estudio sobre revistas españolas incluidas en el índice de revistas de Ciencias Sociales In-Recs (Autor, 2012b). 


\section{Metodología}

Por lo expuesto, el problema se centrará en responder la siguiente cuestión: ¿Cuáles son los descriptores básicos que caracterizan a los artículos científicos sobre Educación Matemática?. Si bien el problema lo enfocamos a la EMA, el proceso metodológico que seguiremos puede ser aplicable a otras disciplinas.

La población objeto de nuestro estudio está formada por todas las publicaciones sobre investigación en Educación Matemática publicadas en revistas científicas entre enero de 1980 y diciembre de 2009 . Se ha tomado este rango de años con la intención de cubrir una distancia de tiempo suficientemente amplio como para poder analizar una amplia gama de artículos y por tanto generar unos descriptores que no respondan sólo a frentes de investigación temporales. Dado que es imposible considerar todas las publicaciones científicas que traten temas sobre EMA, se tomará una muestra representativa de las mismas. Los motivos de esta imposibilidad son entre otros:

- Excesivo número de revistas científicas que podrían publicar artículos de Educación Matemática, en parte debido a que la EMA como ciencia tiene origen en disciplinas muy diversas recibiendo aportes de todas ellas. Lo que hace inmanejable tal volumen de información.

- La no existencia de un tesauro (lista cerrada de descriptores) específico de Educación Matemática.

- La subjetividad que siempre se dará al categorizar las publicaciones científicas como de EMA.

De modo que nuestra muestra la obtendremos de las bases de datos Social Sciences Citation Index (SSCI) de la WoS y SCOPUS. Descargaremos los datos de todos los registros correspondientes a artículos de investigación consideramos como de EMA y que hayan sido publicados en revistas indexadas en estas bases de datos.

A continuación detallamos el proceso de selección de los descriptores que nos permitan recuperar los artículos de investigación en EMA a nivel internacional (Figura 1). Este proceso sigue el esquema propuesto por Medina-López, Marín-García y Alfalla-Luque (2010):

1. Identificación del campo de estudio y periodo a analizar: Investigación en Educación Matemática, 1980-2009.

2. Selección de las fuentes: artículos de investigación.

3. Realización de la búsqueda: Búsqueda automática (WoS y SCOPUS) con el listado básico de descriptores finales obtenidos mediante el proceso que detallamos a lo largo de este apartado.

4. Gestión y depuración de los resultados:

a) Prueba piloto para el filtrado de los resultados.

b) Recuperación de los artículos de Educación Matemática en nuestro periodo de estudio.

5. Análisis de los resultados. 


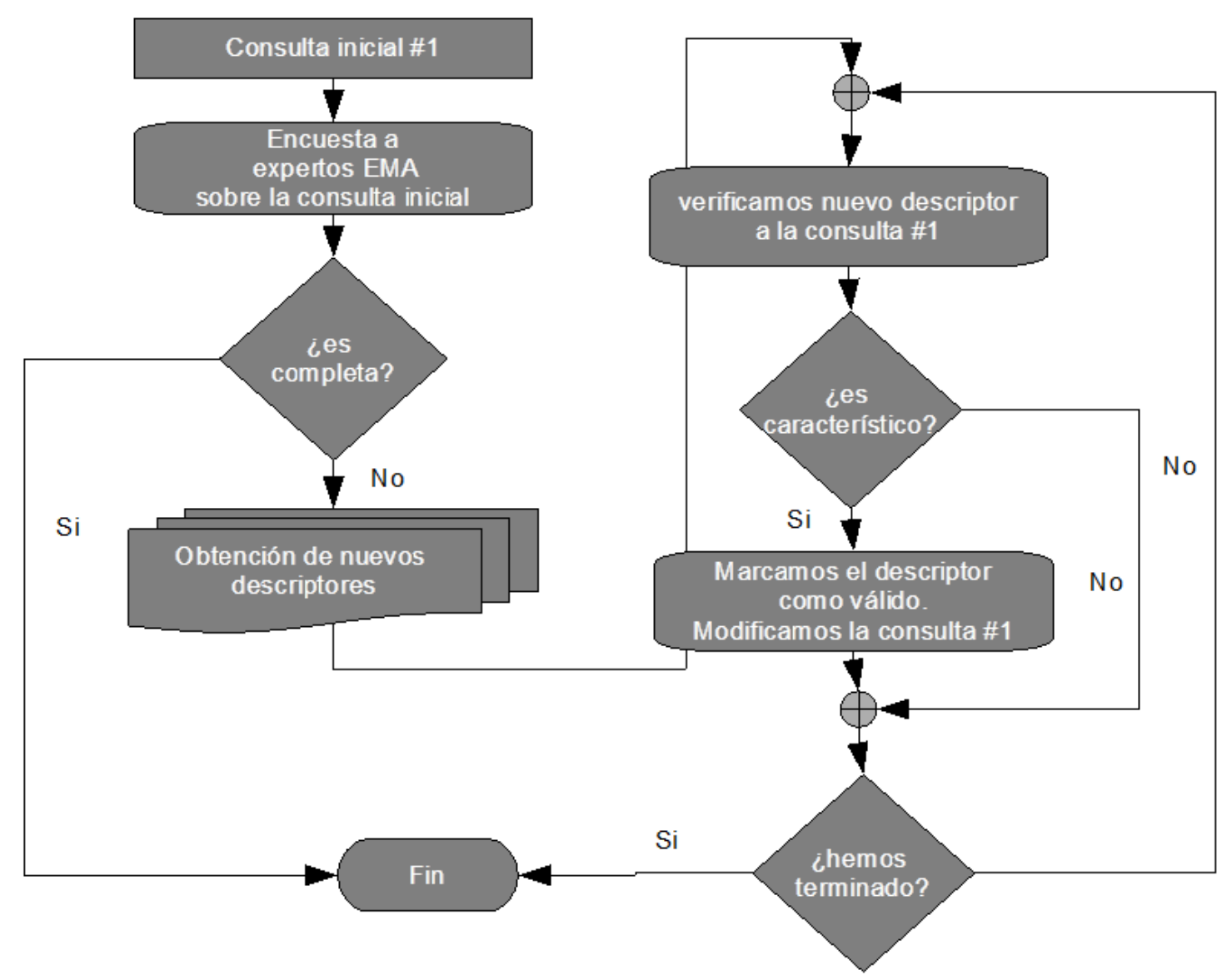

Figura 1. Proceso de elaboración del listado de descriptores

\section{Resultados}

Siguiendo el proceso mostrado (Figura 1), en primer lugar se elaboró un listado de descriptores los cuales fueron propuestos en la consulta realizada a 10 expertos del área (Catedráticos de Didáctica de la Matemática) para su evaluación y posterior modificación y/o ampliación (Tabla I).

Tabla I. Consulta inicial remitida a los expertos de EMA (consulta inicial \#1)

\section{Mathematic*}

ligada a alguna de las siguientes palabras clave:

curricul $^{*}$, instruc ${ }^{*}$, history, educ*, learn ${ }^{*}$, texbooks, teach ${ }^{*}$, Assessment, Didactics,

School, AIDS, student*

Fuente: elaboración propia. 
Los expertos consultados indicaron que la consulta inicial \#1 era válida aunque incompleta, por lo que cada uno sugirió algunos términos más (Tabla II).

Tabla II. Nuevos descriptores de EMA añadidos

\begin{tabular}{|l|l|l|}
\hline \multicolumn{2}{|l|}{ Descriptores } \\
\hline Problem solving & Calculus & Number \\
\hline Technology & CAS & Opportunities to learn \\
\hline Attitudes & Cognition and Affect & Procedures \\
\hline Class & Communication & Professional knowledge \\
\hline Classroom behaviour & Competence & Proof \\
\hline Cognitive & Conceptions & Qualitative \\
\hline Epistemology & Learning difficulties & Research \\
\hline Geometry & Manipulatives & Secondary \\
\hline Primary & Manuales & $\begin{array}{l}\text { Secondary prospective mathamtics } \\
\text { teacher }\end{array}$ \\
\hline Achievement & Mathematical goals & Skills \\
\hline Affective domain & Mathematical knowledge & Standars \\
\hline Algebra & Mathematical modelling & Theory \\
\hline Arithmetic & Methodology & Understanding \\
\hline Believes & Motivation & University \\
\hline
\end{tabular}

Fuente: elaboración propia.

Se establecieron dos grandes grupos de categorias para organizar las combinaciones de los descriptores en las consultas y así poder evaluar su pertinencia, a saber:

a) Descriptores propios de Matemáticas: mathemat*, Algebra, Analysis, Arithmetic, Calculus, Discrete mathematic, Geometry, Number, Probability, Statistic, Topology, Applied mathematic

\section{b) Descriptores propios de Educación:}

- curricul $^{*}$, instruc*, history, educ*, learn*, teach*, Assessment, Didactics, School, student*, class, methodology

- Primary, secondary, university, Preschool, kindergarden

- Attitudes, classroom behaviour, cognitive, affective domain, believes, Cognition and Affect, communication, conceptions, learning difficulties, motivation, Epistemology, competence, Achievement

- opportunities to learn, procedures, professional knowledge, proof, qualitative, research, Secondary prospective mathematics teacher, skills, standars, theory, understanding

- technology, CAS, manipulatives, manuales, aids, texbooks

- $\quad$ problem solving, mathematical goals, mathematical knowlodge, mathematical modelling 
Para la validación de los descriptores, se comenzó por una combinación pequeña de los mismos constituida por la consulta inicial \#1 más algunos de los descriptores sugeridos, que denominamos consulta base (ver Tabla III).

\section{Tabla III. Consulta base}

(mathemat* OR algebra OR Analysis OR arithmetic OR calculus OR Discrete mathematic OR geometry OR number OR Probability OR statistic OR Topology OR Applied mathematic)

AND

(curricul* OR instruc* OR history OR educ* OR learn* OR texbooks OR teach*OR Assessment OR Didactics OR School OR AIDS OR student*)

Fuente: elaboración propia.

Esta consulta base se fue ampliando progresivamente, de forma sistemática y cíclica, añadiendo descriptores nuevos y analizando las nuevas aportaciones debidas exclusivamente a dichos descriptores, siguiendo el diagrama de flujo de la Figura 1.

Este proceso se lleva a cabo de la siguiente forma:

1. Se accede a las bases de datos WoS y SCOPUS a través del portal de la universidad.

2. Para WoS se introducen las combinaciones de las palabras clave o descriptores en los campos de título, resumen y palabras clave. Esto es, el campo Topic. Los operadores booleanos utilizados para combinar las palabras clave son: AND, OR y NOT. En SCOPUS se ponen los descriptores en los campos de título, resumen y palabras clave. Esto es, el campo "TITLE-ABS-KEY".

3. No se establece rango de tiempo para la validación de los descriptores, aunque sí para las consultas piloto y para la descarga de la muestra. Esto es debido a que queremos obtener un listado de descriptores que nos caractericen cualquier artículo de investigación como de Educación Matemática, independientemente del año de publicación.

4. En ambas bases se selecciona como restricción que el tipo de documento sea artículo y que pertenezca, en WoS al área de Socials Sciences Citation Index (SSCI) y en SCOPUS se elige Social Sciences \& Humanities.

Como se observa, la consulta (Tabla III) liga la aparición de cualquiera de los descriptores propuestos propios de Matemáticas (primera fila) a cualquier descriptor propio de Educación (segunda fila).

La primera consulta en WoS y SCOPUS arrojaron más de 100.000 registros en cada una. Se detectó mucho ruido, se realizaron diferentes filtrados con la opción refine results en ambas bases de datos lo que permitió identificar los términos sospechosos de introducir ruido. Posteriormente, se realizó una consulta exclusiva de estos términos (\#A) que combinada con la consulta inicial (\#B) determinó cuáles eran los artículos que se introducen exclusivamente debido a este término sospechoso. Esta consulta combinada (\#A NOT \#B) nos permite evaluar si esas aportaciones nuevas son realmente artículos de Educación Matemática o no, esto es, si el término sospechoso introduce ruido y por lo tanto debe ser finalmente descartado. Con este proceso se descartan tres descriptores propios de Matemáticas, a saber: analysis, calculus, number. Estos términos son muy genéricos y por tanto ligados a cualquier término clave propio de educación, nos introducía en los resultados artículos que no son de Educación Matemática.

Mediante este mismo procedimiento, se excluyeron los siguientes términos por los motivos que a continuación se indican: 
a) Discrete mathematic y Applied mathematic. Por estar incluidos en el descriptor genérico mathemat*, es decir, por no aportar ningún artículo EMA nuevo.

b) Statistic y Probability. Debido a que el campo de la Educación en Estadística utiliza otros descriptores específicos distintos a los usualmente utilizados para Educación Matemática (Ortiz, 2010).

De esta forma, queda configurada la nueva consulta base que se muestra en la Tabla IV, y que arrojó un total de 9.658 artículos en WoS y 20.069 en SCOPUS.

\section{Tabla IV. Consulta base \#2}

(mathemat* OR algebra OR arithmetic OR geometry OR Topology)

\section{AND}

(curricul* OR instruc* OR history OR educ* OR learn* OR texbooks OR teach* OR

Assessment OR Didactics OR School OR AIDS OR student*)

Fuente: elaboración propia.

Tras un proceso similar al realizado anteriormente se detecta el descriptor que introduce el ruido, este es, AIDS (recursos), porque coincide con las siglas en ingles del SIDA.

De igual forma se procede con el término school, pero con resultados diferentes puesto que a pesar de introducir ruido, aproximadamente un $50 \%$ de un total de 615 , este término caracteriza, además de manera exclusiva, a numerosos artículos de EMA que de otra forma no serían detectados. Se comenzó a añadir a la segunda tanda de descriptores de la consulta base \#2 (Tabla V), de descriptores específicos de Educación, nuevos términos para su validación.

\section{Tabla V. Nueva consulta base (consulta \#3)}

(mathemat ${ }^{*}$ OR algebra OR arithmetic OR geometry OR Topology)

\section{AND}

(curricul* OR instruc* OR history OR educ* OR learn* OR texbooks OR teach* OR

Assessment OR Didactics OR School OR student*)

\section{Fuente: elaboración propia.}

Un caso de especial interés es el del descriptor textbooks que no introduce ningún artículo nuevo de EMA, es decir, constituye un término superfluo debido a que en todos los artículos en los que aparece, lo hace ligado a otros descriptores ya tenidos en cuenta. Se decide por tanto su exclusión. Se continuó probando otros descriptores, de los cuales unos fueron rechazados o excluidos de la consulta, tal es el caso de mathematical modelling, classroom behaviour, kindergarten; y otros aceptados, como por ejemplo assessment, class. Así, se obtuvo la consulta base final sobre la que se realizó la prueba piloto (Tabla VI): 
Tabla VI. Nueva consulta base (consulta \#4)

(mathemat* OR algebra OR arithmetic OR geometry OR Topology)

AND

(curricul* OR instruc* OR history OR educ* OR learn* OR teach* OR Assessment

OR Didactic* OR School* OR student* OR goal* OR knowledge* OR classroom*

OR achievement OR competen* OR "problem solving" OR skill* OR PISA OR

TIMSS OR dyscalculia)

Fuente: elaboración propia.

\section{Validación mediante prueba piloto}

Una vez obtenido nuestra tentativa del listado definitivo de descriptores, el siguiente paso fue realizar una prueba piloto en un periodo de cuatro años repartidos dentro del rango de nuestro estudio para que esta muestra sea representativa de la población a lo largo del periodo de años estudiado. Así, los años escogidos para la prueba piloto fueron: 1980, 1989, 1999 y 2009.

Esto nos arrojó un total de 1646 artículos en WoS y 3641 en SCOPUS, como nos pareció una muestra demasiado amplia para revisar uno a uno, decidimos tomar, mediante muestreo aleatorio estratificado, la mitad de registros de cada año. De esta forma nuestra prueba piloto la conforman 824 artículos en WoS y 1820 en SCOPUS.

El análisis de los resultados arrojados por la base de datos en esta prueba piloto fue manual, analizando los artículos (Título, palabras clave y resumen) y clasificando dichos artículos como de Educación Matemática, no Educación Matemática o dudosos. Para la clasificación final de los artículos dudosos se realizó un análisis más exhaustivo acudiendo a la fuente completa en la mayoría de los casos y recurriendo a la triangulación por pares. De este análisis se obtuvieron los datos reflejados en la tabla VII.

Tabla VII. Clasificación de artículos EMA en muestra piloto

\begin{tabular}{|l|r|r|r|r|c|}
\hline & WoS & \multicolumn{1}{|c|}{$\%$} & \multicolumn{1}{c|}{ ScOPUS } & \multicolumn{1}{c|}{ Total } & \\
\hline Artículos descargados & 1646 & 31,13 & 3641 & 68,87 & 5287 \\
\hline Muestra reducida a la mitad & 824 & 31,16 & 1820 & 68,84 & 2644 \\
\hline Artículos de EMA & 486 & 36,43 & 848 & 63,57 & 1334 \\
\hline Artículos que no son de EMA & 338 & 25,80 & 972 & 74,20 & 1310 \\
\hline
\end{tabular}

Fuente: elaboración propia.

De esta clasificación se observa que el porcentaje de ruido o falsos positivos en la muestra reducida, asciende al $41,02 \%$ (WoS) y al $53,41 \%$ (SCOPUS) del total en cada base de datos por lo que podemos optar por dos soluciones:

a) Modificar la estrategia de búsqueda. Esto es, ver la conveniencia de modificar nuestro listado de descriptores EMA y/o la combinación de los mismos.

b) Filtrar la muestra descargada en base a unos criterios establecidos. 
Tras diversas pruebas mediante la modificación de la estrategia de búsqueda, descartamos esta opción debido al aumento de falsos negativos, esto es, artículos que tenemos la certeza que son de EMA y son excluidos de la búsqueda. Debido a esto, nos decantamos por la opción b0, dando por válida la estrategia de búsqueda y por tanto nuestro listado tentativo de descriptores de Educación Matemática. Se trata entonces de obtener un filtrado óptimo, que reduzca el ruido a un porcentaje asumible, de manera que no excluya prácticamente ningún artículo de EMA y elimine prácticamente todos los artículos que no son de EMA. Una vez tengamos bien definidos los criterios de filtrado, se aplicaran a la muestra completa.

Para el filtrado, realizamos un análisis de los nombres de las revistas que tienen artículos que habíamos catalogado como de EMA, por un lado, y por otro los títulos de las revistas que tienen artículos que no son de Educación matemática. Calculamos la frecuencia de artículos en ambos casos. Cruzando ambas tablas obtenemos 209 revistas en WoS y 369 en SCOPUS que exclusivamente publican artículos no clasificados como de EMA en nuestra muestra piloto (Erro! Fonte de referência não encontrada.VIII), es decir, revistas que exclusivamente introducen ruido en la muestra (sombreadas en la tabla). Estas publicaciones las marcamos e incluimos en un listado de revistas a excluir específico para cada base de datos.

Tabla VIII. Las 15 primeras revistas WoS y SCOPUS que publican exclusivamente artículos "no EMA"

\begin{tabular}{|c|c|c|c|}
\hline \multicolumn{2}{|l|}{ Wos } & \multicolumn{2}{|l|}{ SCOPUS } \\
\hline Nombre de la Publicación & № artículos & Nombre de la Publicación & № artículos \\
\hline Computers \& Industrial Engineering & 4 & $\begin{array}{l}\text { European Journal of Operational } \\
\text { Research }\end{array}$ & 65 \\
\hline Risk Analysis & 4 & $\begin{array}{l}\text { International Journal of Production } \\
\text { Research }\end{array}$ & 16 \\
\hline Actual Problems Of Economics & 3 & Information sciences & 15 \\
\hline Animal Cognition & 2 & Risk Análisis & 13 \\
\hline Artificial Intelligence & 2 & $\begin{array}{l}\text { International Journal of Production } \\
\text { Economics }\end{array}$ & 12 \\
\hline Cognitive Psychology & 2 & $\begin{array}{l}\text { Mathematics and Computers in } \\
\text { Simulation }\end{array}$ & 11 \\
\hline Child Abuse \& Neglect & 2 & Building and Environment & 9 \\
\hline Ergonomics & 2 & Operations Research & 9 \\
\hline European Journal Of Operational Research & 2 & $\begin{array}{l}\text { Computers and Industrial } \\
\text { Engineering }\end{array}$ & 8 \\
\hline Expert Systems With Applications & 2 & $\begin{array}{l}\text { International Journal of Systems } \\
\text { Science }\end{array}$ & 8 \\
\hline Geographical Analysis & 2 & Nature & 8 \\
\hline International Journal Of Engineering Education & 2 & Production Planning and Control & 8 \\
\hline Information Sciences & 2 & Management Science & 6 \\
\hline $\begin{array}{l}\text { International Journal Of Geographical Information } \\
\text { Science }\end{array}$ & 2 & Safety Science & 6 \\
\hline International Journal Of Industrial Ergonomics & 2 & Speech Communication & 6 \\
\hline
\end{tabular}

Fuente: elaboración propia. 
Del cruce de tablas anterior, obtenemos un listado de títulos de revistas que publican tanto artículos EMA como no EMA, indicando la frecuencia de los mismos. Aplicamos el siguiente criterio: marcar como revistas a excluir las revistas que publiquen más o igual número de artículos no EMA que EMA, en total 35 en WoS y 66 en SCOPUS. La tabla IX ejemplifica este proceso para SCOPUS. De este procedimiento obtenemos un listado de 244 títulos de publicaciones a excluir en WoS y 435 en SCOPUS.

Tabla IX. Las 15 primeras revistas SCOPUS con mayor porcentaje de artículos "no EMA" publicados

\begin{tabular}{|c|c|c|c|}
\hline \multicolumn{4}{|c|}{ Wos } \\
\hline Nombre de la Publicación & $\begin{array}{c}\text { № Art. } \\
\text { EMA }\end{array}$ & $\begin{array}{l}\text { № Art. } \\
\text { № EMA }\end{array}$ & $\begin{array}{c}\% \\
\text { No EMA }\end{array}$ \\
\hline Computers and Operations Research & 1 & 19 & 95,00 \\
\hline Journal of the Operational Research Society & 1 & 14 & 93,33 \\
\hline Mathematical and Computer Modelling & 1 & 9 & 90,00 \\
\hline $\begin{array}{l}\text { Proceedings of the National Academy of Sciences of the } \\
\text { United States of America }\end{array}$ & 3 & 22 & 88,00 \\
\hline Accident Analysis and Prevention & 1 & 7 & 87,50 \\
\hline Journal of Statistics Education & 1 & 6 & 85,71 \\
\hline Synthese & 1 & 6 & 85,71 \\
\hline Psychonomic Bulletin and Review & 1 & 5 & 83,33 \\
\hline IEEE Transactions on Education & 3 & 14 & 82,35 \\
\hline Cognitive Systems Research & 1 & 4 & 80,00 \\
\hline Foundations of Science & 1 & 4 & 80,00 \\
\hline Journal of Clinical and Experimental Neuropsychology & 1 & 4 & 80,00 \\
\hline Journal of Studies on Alcohol and Drugs & 1 & 3 & 75,00 \\
\hline International Journal of Electrical Engineering Education & 2 & 5 & 71,43 \\
\hline Computers in Human Behavior & 2 & 4 & 66,67 \\
\hline
\end{tabular}

Fuente: elaboración propia.

En nuestra base de datos ad hoc, se eliminan los artículos que pertenecen a las revistas incluidas en el listado de revistas a excluir, obteniendo 486 registros válidos en WoS y 823 en SCOPUS (Tabla 9). Detallamos en la tabla X los porcentajes de artículos EMA y no EMA en el conjunto de artículos aceptados y rechazados tras el filtrado. 
Bílios Search for descriptors that characterize an emerging discipline in WoS...

Tabla X. Porcentajes de artículos WoS de EMA y no EMA en el conjunto de artículos aceptados y no aceptados tras el filtrado

\begin{tabular}{|c|c|c|c|c|c|c|}
\hline & \multicolumn{2}{|c|}{ Número de Artículos } & \multicolumn{2}{|c|}{$\begin{array}{l}\% \text { sobre la Muestra total reducida } \\
\text { a mitad }\end{array}$} & \multicolumn{2}{|c|}{ \% sobre los aceptados } \\
\hline & Wos & SCOPUS & $\begin{array}{l}\text { WoS } \\
\text { (824) }\end{array}$ & $\begin{array}{c}\text { SCOPUS } \\
(1820)\end{array}$ & $\begin{array}{l}\text { WoS } \\
(486)\end{array}$ & $\begin{array}{c}\text { SCOPUS } \\
\text { (823) }\end{array}$ \\
\hline \multicolumn{7}{|c|}{ Aceptados } \\
\hline no EMA & 48 & 59 & 5,83 & 3,24 & 9,88 & 7,17 \\
\hline EMA & 438 & 764 & 53,16 & 41,98 & 90,12 & 92,84 \\
\hline \multicolumn{7}{|c|}{ No aceptados } \\
\hline no EMA & 293 & 913 & 35,55 & 50,16 & 86,68 & 91,57 \\
\hline EMA & 45 & 84 & 5,46 & 4,61 & 13,31 & 8,43 \\
\hline
\end{tabular}

En vista de los resultados, podemos considerar que el filtrado realizado es pertinente y depura la muestra, tal y como pretendíamos. En la tabla XI se presenta el listado definitivo de descriptores que van a definir un artículo como de Educación Matemática en WoS y SCOPUS.

\section{Obtención de la muestra final}

Una vez realizada la validación mediante el filtrado de la prueba piloto, ya estamos en condiciones de aplicar el filtrado realizado a nuestra muestra total de artículos descargados de WoS y SCOPUS.

La descarga se realizó el día 13 de octubre de 2011. De la misma resultaron un total de 37152 artículos, correspondiendo 10347 a WoS y 26805 a SCOPUS. A este conjunto de artículos le aplicamos el filtrado de títulos de revistas en nuestra base ad hoc, descartando 2047 artículos de WoS y 13400 de SCOPUS, reduciéndose la muestra final de trabajo a 21705 artículos. 
Tabla XI. Descriptores que caracterizan la Educación Matemática en WoS y SCOPUS

\begin{tabular}{|c|c|}
\hline \multicolumn{2}{|c|}{ Descriptores de Educación Matemática } \\
\hline $\begin{array}{l} \\
\text { Mathemat* } \\
\text { Algebra } \\
\text { Arthmetic } \\
\text { Geometry } \\
\text { Topology }\end{array}$ & $\begin{array}{l}\text { Curricul* } \\
\text { Instruc* } \\
\text { History } \\
\text { Educ* } \\
\text { Learn* }^{*} \\
\text { Teach* } \\
\text { Assessment } \\
\text { Didactic* } \\
\text { School* } \\
\text { student* } \\
\text { Goal* } \\
\text { Knowledge* } \\
\text { classroom* } \\
\text { Achievement } \\
\text { Competen* } \\
\text { "Problem solving" } \\
\text { Skill* } \\
\text { PISA } \\
\text { TIMSS } \\
\text { Dyscalculia }\end{array}$ \\
\hline
\end{tabular}

Fuente: elaboración propia.

\section{Conclusiones}

Hemos hallado una serie de descriptores que caracterizan los artículos de investigación en Educación Matemática. Estos nos ha permitido rescatar de dos bases de datos internacionales, como lo son WoS y SCOPUS, prácticamente todos los artículos de investigación en Educación Matemática que allí se indizan. Por supuesto, la gran importancia de este listado es que va a permitir obtener la producción de esta área de conocimiento en cualquier otra base de datos.

Hemos descrito un proceso metodológico para caracterizar una disciplina científica que se encuentra en un cruce de diversas ciencias (Educación, Psicología, Matemáticas, etc.) lo que dificultaba en un principio la búsqueda de artículos de investigación en la materia publicados en revistas no específicas de la disciplina. Este mismo proceso para la obtención de los descriptores puede ser aplicado a otras disciplinas emergentes que comparten múltiples puntos de conexión y relación con otras ramas científicas, como lo son las didácticas específicas. 
Aplicando este proceso se obtienen descriptores básicos confiables para obtener información de las bases de datos y poder así realizar estudios bibliométricos y temáticos con un mayor volumen de información sobre disciplinas emergentes y que no cuentan con un número destacado de revistas internacionales especificas con presencia en las bases de datos internacionales y por tanto sus producciones se difunden en revistas generalistas o especificas de las ciencias con las que comparten algún tipo de relación temática, conceptual, epistemológica o teórica.

La importancia de contar con métodos que permitan obtener los descriptores relevantes de disciplinas que no cuentan con tesauros específicos, como el aquí descrito, radica en que permite realizar estudios bibliométricos de la producción científica y de las redes académicas que generan, para identificar tendencias, grupos, escuelas y agendas de investigación de estas disciplinas.

\section{Bibliografía}

Alcain, M. D. y Ruíz-Gálvez, M. (1996). Bases de datos y repertorios bibliográficos. Papeles del Psicólogo, 64, 1-11

Barité, M. (2009). Garantía literaria y normas para construcción de vocabularios controlados: aspectos epistemológicos y metodológicos. Scire, 15(2), 13-24.

Granados, M. y Nicolau, A. (2007). Aplicación de un nuevo sistema de indización en una colección de recursos especializados en ciencias de la educación. El Profesional de la Información, 16(6), 627-235.

Godino, J. D. (1991). Hacia una teoría de la educación matemática. En Gutiérez, A. (ed.), Área de conocimiento: Didáctica de la matemática (pp. 77-105). Madrid: Síntesis.

Marín, N. (2010). Propuesta para compartir una base de datos de información bibliográfica (BIB) en didáctica de las ciencias. Rev. Eureka Enseñ. Divul. Cien., 7(3), 613-635.

Autor (2003). Análisis cienciométrico de las tesis doctorales españolas en Educación Matemática. Revista española de documentación científica, 26(2), 162-176.

Autor (2009). La Educación Matemática en la revista Enseñanza de las Ciencias: 1983-2006. Enseñanza de las Ciencias , 27 (2), 185-194.

Autor (2011). La investigación en Educación Matemática en España: los simposios de la SEIEM. PNA, 5 (4), 163-184.

Autor (2012a). Redes académicas generadas por las tesis doctorales de educación matemática en España. Revista e Investigación Educativa, 30(2).

Autor (2012b). La investigación en Educación Matemática a través de las publicaciones científicas españolas. Revista Española de Documentación Científica, 35 (2), 262-280 (2012)

Medina-López, C., Marín-García, J. A. y Alfalla-Luque, R. (2010). A methodological proposal for the systematic literature review. WPOM-Working Papers on Operations Management, 1(2), 13-30.

Quintarelli, E. (2008). Folksonomies: power to the people. En: ISKO Italy-UniMIB Meeting, 2005, Milán. Disponible en: http://www.iskoi.org/doc/folksonomies.htm

Ortiz, J. J. (2010). La educación estadística en los Simposios de la SEIEM (1997-2009). In M. M. Moreno, A. Estrada, J. Carrillo \& T. A. Sierra (Eds.), Investigación en Educación Matemática XIV (pp. 475-486). Lleida: SEIEM.

Rodríguez-Yuste, L. (2009). Etiquetado libre frente a lenguajes documentales. Aportaciones en el ámbito de biblioteconomía y documentación. En: IX Congreso ISKO-España. Nuevas perspectivas para la difusión y organización del conocimiento. Actas del Congreso. Valencia: UPV, vol 2, pp. 832-845.

Sanz-Valero, J., Veiga, J., Rojo-Alonso, C., D’Agostino, M. J., Wanden-Berghe, C., Espulgues, J. X. y Rodrigues, C. (2008). Los filtros metodológicos: aplicación a la Búsqueda bibliográfica en la medicina del trabajo española. Med Segur Trab, LIV(211), 75-83.

Senso, J. A., Magaña-Redondo, P. J., Faber-Benitez, P. y Vila-Miranda, A. (2007). Metodología para la estructuración del conocimiento de una disciplina: el caso de PuertoTerm. El Profesional de la Información, 16(6), 591.604.

Strehl, L. (2011). As folksonomias entre os conceitos e os pontos de acesso: as funções de descritores, citações e marcadores nos sistemas de recuperação da informação. Perspectivas em Ciência da Informação, 16, 101-114. 


\section{Datos de los autores}

Natividad Adamuz-Povedano

Profesora del Departamento de Matemáticas, Universidad de Córdoba.

lf2adpon@uco.es

Noelia Jiménez-Fanjul

Profesora del Departamento de Matemáticas, Universidad de Córdoba.

noelia.jimenez@uco.es

Alexander Maz-Machado

Profesor del Departamento de Matemáticas, Universidad de Córdoba.

ma1mamaa@uco.es

Recibido-Received : 2013-02-18

Aceptado-Accepted : 2013-03-30 
Biblios search for descriptors that characterize an emerging discipline in WoS...

(cc) $\mathbf{E Y}$ New articles in this journal are licensed under a Creative Commons Attribution 3.0 United States License.

UILIS D-Sult

This journal is published by the University Library System of the University of Pittsburgh as part of its D-Scribe Digital Publishing Program and is cosponsored by the University of Pittsburgh Press. 